\title{
Associations between ideational variables and bed net use in Madagascar, Mali, and Nigeria
}

\author{
J. Douglas Storey* ${ }^{*}$, Stella O. Babalola, Emily E. Ricotta, Kathleen A. Fox, Michael Toso, Nan Lewicky
} and Hannah Koenker

\begin{abstract}
Background: The use of insecticide-treated bed nets (ITNs) is crucial to the prevention, control, and elimination of malaria. Using household surveys conducted in 2014-2015 by the Health Communication Capacity Collaborative project in Madagascar, Mali, and Nigeria, we compared a model of psychosocial influence, called Ideation, to examine how malaria-related variables influence individual and household bed net use in each of these countries. Evaluations of non-malaria programs have confirmed the value of the ideational approach, but it is infrequently used to guide malaria interventions. The study objective was to examine how well this model could identify potentially effective malaria prevention approaches in different contexts.
\end{abstract}

Methods: Sampling and survey designs were similar across countries. A multi-stage random sampling process selected female caregivers with at least one child under 5 years of age for interviews. Additional data were collected from household heads about bed net use and other characteristics of household members. The caregiver survey measured psychosocial variables that were subjected to bivariate and multivariate analysis to identify significant ideational variables related to bed net use.

Results: In all three countries, children and adolescents over five were less likely to sleep under a net compared to children under five ( $\mathrm{OR}=0.441$ in Madagascar, 0.332 in Mali, 0.502 in Nigeria). Adults were less likely to sleep under a net compared to children under five in Mali $(O R=0.374)$ and Nigeria $(O R=0.448)$, but not Madagascar. In all countries, the odds of bed net use were lower in larger compared to smaller households $(\mathrm{OR}=0.452$ in Madagascar and $\mathrm{OR}=0$. 529 in Nigeria for households with 5 or 6 members compared to those with less than 5; and $O R=0.831$ in Mali for larger compared to smaller households). Of 14 common ideational variables examined in this study, six were significant predictors in Madagascar (all positive), three in Mali (all positive), and two in Nigeria (both negative).

Conclusion: This research suggests that the systematic use of this model to identify relevant ideational variables in a particular setting can guide the development of communication strategies and messaging, thereby improving the effectiveness of malaria prevention and control.

Keywords: Malaria, Ideation, Bed nets, Insecticide treated nets, Health communication, Behavior change

\footnotetext{
* Correspondence: dstorey@jhu.edu

Johns Hopkins Center for Communication Programs, 111 Market Place, Suite

310, Baltimore, MD 21202, USA
} 


\section{Background}

As of 2016, sub-Saharan Africa carries the vast majority of the global burden of malaria cases (90\%) and deaths (92\%); $70 \%$ of the deaths were in children under five [1]. The use of ITNs has been shown to reduce malaria incidence rates by $50 \%$ in children under five [1] and mass ITN distribution campaigns and routine distribution channels have been shown to be effective and costefficient ways of increasing household ITN access and reducing malaria morbidity and mortality [2-7]. However, while access to nets remains a barrier to achieving consistent net use in some places [4, 8-10], the question persists of how to ensure that people obtain and use the nets to which they have access.

At a systems level, access to bed nets is ensured by a country's ability to provide bed nets through mass or continuous distribution, and retail or social marketing channels to achieve universal coverage [4]. The standard Roll Back Malaria indicator of population access to ITN refers to the proportion of individuals that could use a net within a household, assuming one net covers two people. An additional standard indicator is the proportion of households that own enough ITNs to achieve the World Health Organization's universal coverage recommendation of one ITN for every two people $[4,8,9,11]$. Recent reports indicate that ITN use and access are strongly correlated $[10,12]$. Over several recent MIS and DHS surveys, Madagascar and Mali both have very high ratios of ITN use to ITN access ( $>1.00$, indicating more than 2 people on average share an ITN, and $>0.90$, respectively), while Nigeria's 2015 MIS documented a ratio of 0.68 . In all three countries, the ratio varies at the subnational level and by socioeconomic status and residence [10]. In the few countries where access and use are not strongly correlated, and among groups of people with access who do not use ITNs, social and behavior change communication (SBCC) campaigns offer the most feasible means of closing the ITN access/use gap [10, 12].

SBCC campaigns can help to change or reinforce behaviors necessary to obtain, and/or maintain the appropriate number of ITNs in a household, as well as sleep under a bed net [4, 13-16] by focusing on particular psychosocial determinants of behavior. Most behaviors are not driven by a single variable or determinant. For example, preventive health behaviors do not result from a fear of disease alone, but are also-often simultaneously-influenced by such things as concerns about the cost or inconvenience of protective measures, confidence or doubts about the effectiveness of a treatment, and motivation to do what others in the community do [17]. In this study, we conducted a comparison of a particular model of psychosocial influence, known as the Ideation Model of Strategic Communication and Behavior Change $[4,18-20]$, to examine how malaria-related ideational variables influence individual and household bed net use in each of these countries. Evaluations of programs on other health topics have confirmed the predictive value of the ideational approach, but it has not been widely used to guide malaria interventions. The main objective of this study was to determine the degree to which the ideation model was generalizable as a guide to identifying potentially effective malaria prevention approaches in three different countries.

The ideation model (Fig. 1) is part of a metatheory of strategic communication and behavior change $[19,20]$ that incorporates intermediate cognitive, emotional and social constructs from various behavioral theories and models. Different theories tend to emphasize different factors and variables associated with behavior change. Some emphasize cognitive variables, such as beliefs, values, and attitudes [21-25]; others include emotional variables, such as fear or elation, empathy, and confidence, or self-efficacy [26-28]; while others place more emphasis on conative variables, such as social support, social influence, spousal/partner communication, and personal advocacy [29-35]. These are listed in the central box of Fig. 1. While many SBCC campaign strategies emphasize social and psychological determinants of behavior, the ideation model described here is unique in that it emphasizes three things: (1) an individual's decision-making process leading to a behavioral choice is complex and can involve multiple variables simultaneously, (2) ideational variables are behavior-specific, and (3) the influence of those multiple variables is cumulative, that is, the more of the variables that are positive with regard to the behavior, the higher the probability of that behavior occurring. Furthermore, all ideational variables can be influenced by social interaction and a variety of instructive, directive, non-directive and public communication forms, often through mass media exposure $[4,11,36,37]$, which increases the likelihood of population-level change.

Figure 1 also indicates that the ideational change resulting from communication complements other changes in skills and knowledge necessary to perform a particular action, like bed net use, as well as changes in the environmental context, like socio-economic conditions, policies and types of material and technological support for health improvement efforts. When ideational change occurs and is supported by appropriate knowledge and environmental conditions, the behavior of individuals and groups of people is likely to change, resulting in improved health outcomes. The more ideational factors that improve, the greater the probability of behavior change. This model has been used to help guide and explain the effects of a variety of health behavior programs, including contraceptive adoption [36, 3840], HIV testing [41], HIV prevention [42], child survival 


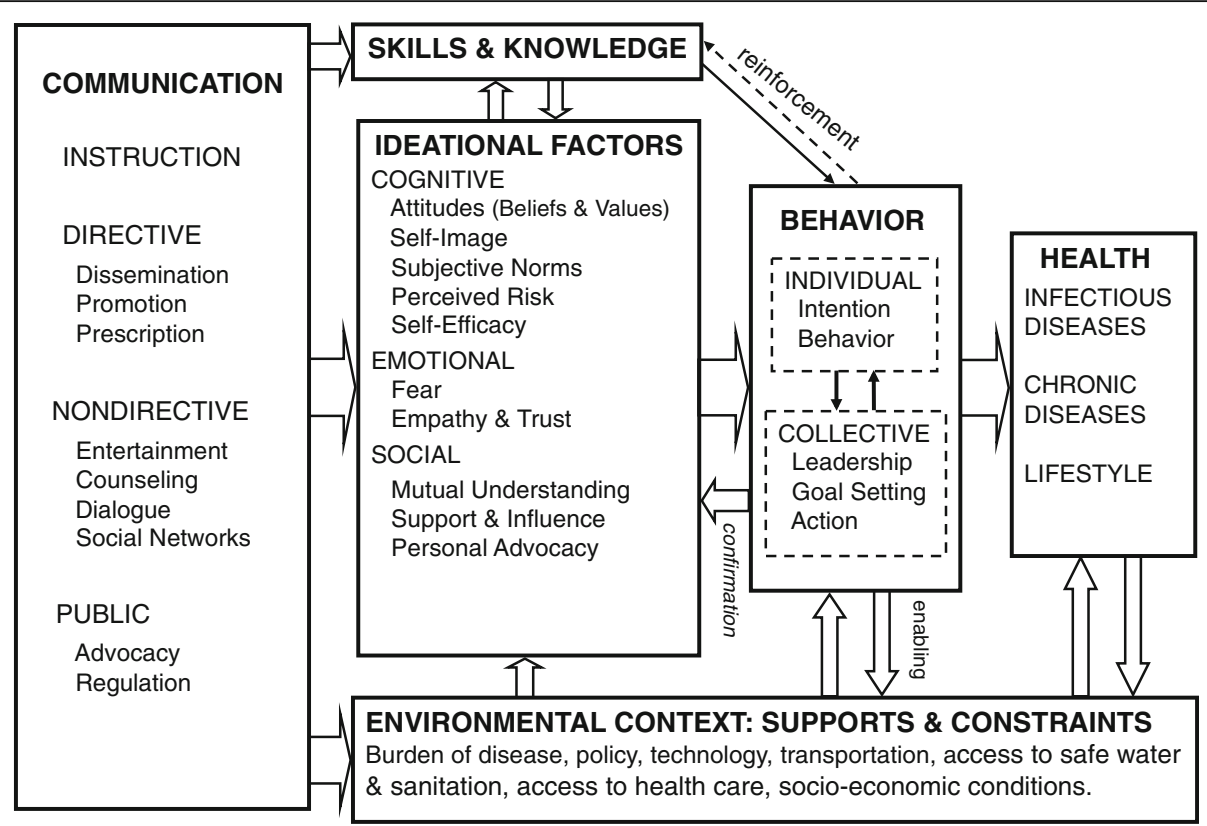

Fig. 1 Ideation model of strategic communication and behavior change. Detailed legend: Ideation model of strategic communication and behavior change showing relationships between types of communication, skills and knowledge, environmental context, ideation, behavior change and health outcomes [73]

[43], household treatment and handling of water [44, 45], community support to reduce girls' vulnerability to HIV/ AIDS [46], and Ebola response [47]. Human-centered design approaches [48] in health and other behavioral domains have begun to use the ideation model to understand customer needs and develop client-oriented products that resonate with those needs.

In the case of malaria, one would not expect the same set of beliefs, attitudes, norms, and perceptions to influence, for example, both bed net use and care seeking for fever. Similarly, one would not expect the same set of ideational variables to influence bed net use both in year-round malaria areas and in areas with highly seasonal transmission, because patterns of risk perception and susceptibility are likely to be quite different, thus underscoring the importance of exploring the ideational variables that are relevant for each behavior and in different settings.

Recently in Tanzania, the first malaria-related study to focus on the ideation process found that a particular set of ideational variables mediated the effects of malaria prevention communication campaigns on householdlevel universal coverage with ITNs [4]. Ideation about bed net use in Tanzania included the following locally specific variables: (1) perceived positive social norms surrounding net ownership and use, (2) belief in one's ability to use nets properly (self-efficacy), (3) belief that net use is an effective way to prevent malaria (sometimes termed 'response' efficacy), and (4) perceived threat of malaria. Exposure to communication campaigns was correlated positively with increased net ideation, that is, the cumulative number of positive ideational variables, which in turn was correlated positively and significantly with household universal ITN coverage, i.e., having enough nets for everyone in the household [4].

The Health Communication Capacity Collaborative (HC3) conducted household surveys in Madagascar, Mali, and Nigeria, to examine the relationships between an individual's net-use behavior and their demographic and psychosocial (ideational) variables. In this article, we compare the ideational variables of bed net use to prevent malaria in these three countries in order to better understand how differences in ideation can be used to inform strategies to improve the effectiveness of malaria prevention and control interventions wherever bed net use is a public health priority.

\section{Methods}

\section{Study setting}

This study is based on analysis of household survey data collected in Madagascar, Mali, and Nigeria between 2014 and 2015 by the Johns Hopkins Center for Communication Programs (CCP), under the United States Agency for International Development (USAID)-funded HC3 project and with the support of the President's Malaria Initiative (PMI). The surveys were designed specifically for the purpose of comparing ideational influences on the use of bed nets for malaria prevention as part of CCP's mandate under this grant. These countries were chosen for the study because they are PMI priority countries. 
CCP designed the questionnaires, but field work was conducted by trained interviewers through a contract with a private research firm in each country, selected through competitive procurement. Questionnaires were translated from the original English into local languages. Depending on respondents' preferences, the interviews were conducted in English, Pidgin English, Igbo or Hausa in Nigeria; French or Bambara in Mali; and Malagasy in Madagascar. Data were collected during the dry season/ early rainy season in Madagascar (between September and November 2014), the rainy season in Mali (between July and September 2015), and the rainy season in Nigeria (between July and September 2015).

The three countries have differing levels of malaria parasitemia in children 6-59 months-measured through microscopy-varying from 9\% in Madagascar [49] to $27 \%$ in Nigeria [50] and 36\% in Mali [51]. While malaria transmission in Nigeria is stable and perennial, northern Sahalian areas experience increases in transmission during 3 months or less of seasonal rains [52]. Mali's Sahalian belt experiences stable, seasonal transmission while in the northern desert region transmission is low, unstable and epidemic-prone [53]. In Madagascar, the sub-desert and highland zones experience shorter seasons of increased transmission, the latter with pockets of high elevation where autochthonous transmission is rare; the tropical and equatorial zones experience stable malaria transmission most of the year [54]. Cyclones occur between December and April and often result in increased risk of malaria infection. The majority of malaria cases are caused by Plasmodium falciparum parasites in all three countries.

Mass bed net distributions took place in each of the three countries prior to survey data collection. While sensitization campaigns accompany most mass distributions, details about SBCC coverage, message exposure, and message recall are only available for a program in Nigeria, namely the Support to the National Malaria Programme (SuNMaP) funded by UKaid, and the NetWorks project funded by the U.S. President's Malaria Initiative [55]. In that instance, over half of respondents in ten different states were exposed to multi-channel SBCC and positive attitudes towards nets were positively associated with the number of messages recalled.

\section{Sampling design and participant characteristics}

The sampling design was similar across the three surveys. Study participants were selected through a multi-stage random sampling process that involved first selecting-with probability proportional to size-districts, local government areas, or communes depending on the country, then clusters or enumeration areas, and finally households with at least one child under 5 years old. In each selected household, a child under 5 years old was randomly selected and the mother of that child was invited to participate in the caregiver survey. In our experience, household heads and caregivers are able to describe not only their own behavior, but that of other members of the family with a fairly high level of reliability. This is especially true for observable behaviors like sleeping under a bed net. We anticipated little additional informational value from multiple interviews in the same household, so opted to interview the caregiver of only one under-five child in each household and distribute the sample across a wider variety of households. In addition, in one third of the selected households, the head of household (if he/she was not the same as the caregiver) was also interviewed to obtain the perspective of the key decision-maker in the household. The multistage sampling design was taken into consideration in calculating the sample size. We applied a design effect of 2.0. Sample weights were not available; so, they were not used in the analyses. The sampling strategy yielded 2390 households in Madagascar, 3202 in Mali, and 3616 in Nigeria. Using a structured household survey questionnaire, information on ownership and use of bed nets was collected on all household members: 12,834 in Madagascar, 19,345 in Mali, and 16,832 in Nigeria. In this manuscript, we merged household-level data with data from the caregiver survey to assess the correlates of household members' net use. Specifically, from the household data, we derived bed net use information and sociodemographic characteristics of individual household members, while data on female caregivers' sociodemographic and ideational variables were derived from the individual caregiver questionnaire.

\section{Variables}

In this study, the dependent variable was whether an individual slept under a bed net on the night before the survey, as reported in the household questionnaire. The variable was derived from a question that asked who slept under each net available, as enumerated in a household net roster, following the Roll Back Malaria Monitoring and Evaluation Working Group's recommendations [56]. Net brand was not recorded consistently, therefore determining a net's status as an ITN was not possible; however, the majority of bed nets in each country are ITNs as observed in recent surveys-94\% in Madagascar in 2013, 97\% in Nigeria in 2015, and 95\% in Mali in 2015 [49-51]. We assessed the predictive value of 25 independent variables measured at the individual, household, and community levels, including household size and the number of nets owned, and the following female caregiver-specific variables:

- education level and religion;

- radio-listening and television-viewing habits;

- exposure to malaria related-information on media or through community sources;

- perceived severity of malaria*; 
- perceived susceptibility to malaria*;

- perceived self-efficacy to prevent malaria*, to detect a severe case of malaria", or to procure enough nets for all members of her household*;

- perceived response efficacy of bed nets";

- knowledge of fever as a symptom of malaria and of mosquitoes as the cause of malaria*;

- discussion of malaria with friends and family during the last 12 months*;

- participation in decisions about net allocation within the household*;

- attitudes toward bed nets";

- perception about bed net use being the norm in their community*;

- awareness of a place to purchase nets"; and

- willingness to pay for nets".

A cumulative ideation score was then calculated as the sum of the number of ideational variables that are positively associated with bed net use in each country (among the items with " above), producing an ideation score.

In addition to the main models that included the individual ideational variables, other models that substituted a composite core-the sum of the number of positive individual ideational variables for each respondent-were tested to assess the Sp: cumulative predictive power of ideation. In addition, we calculated an interaction term between age and sex in the estimated models for all three countries.

\section{Analysis}

We used both bivariate and multivariate analytic methods. The bivariate method compares net use across sociodemographic groups and reports the significance of the differences. Logistic regression was the main multivariable analytic method used and was limited to individuals from households with at least one net and at least one child under 5 years old, representing $73 \%$ of households in Madagascar, 97\% in Mali, and 79\% in Nigeria. We report both the odds ratios and the fullystandardized beta coefficients for the regression models.

\section{Results}

In this section, we report the analysis results for each country separately, then summarize findings across all three countries in the discussion section.

\section{Madagascar}

\section{Bivariate analysis}

Overall, almost three-quarters (73.3\%) of residents of households with at least one net slept under a net. Among these households, net use varied significantly by zone of residence: $39.4 \%$ in the Highlands compared to
71.4\% in Sub-Desert, $72.9 \%$ in Tropical, and $82.3 \%$ in Equatorial $\left(\mathrm{X}^{2}=447.3 ; p<0.001\right)$. Significant differences by age were identified: $79.9 \%$ of children under 5 years old slept under a net compared to $54.5 \%$ among children aged 5-17 years, and $80.1 \%$ of adults $\left(\mathrm{X}^{2}=469.8 ; p<0\right.$. 001). Significant differences by gender were also found, with net use being more common among females (75. $5 \%)$ than among males $(70.9 \%)(\mathrm{z}=4.95 ; p<0.001)$.

\section{Multivariate analysis}

Results of the multivariate logistic regression indicate that the significant demographic predictors of net use among household members are the individual's age, zone of residence, household wealth, household size, and number of nets in the household. Also significant were the female caregiver's television viewing and her level of exposure to malaria-related communication messages. There were no significant interactions between age and gender (Table 1).

Among males, household members aged 5-17 years were $59 \%$ less likely than children under 5 years old to sleep under a net. There was no significant difference in net use between adult males and male children under 5 years old. Among children under 5 years old, gender did not make a difference in the odds of net use. Additionally, being female did not significantly moderate the relationship between age and net use. The relationship of net use and household wealth was such that the only significant difference was found between those in the lowest quintile and their peers in the fourth quintile. The relationship between net use and household size was negative. For example, people from households with nine or more members were $85 \%$ less likely than those from households with fewer than five members. Furthermore, having three or more nets in the household increased the odds of net use almost three-fold compare to having one or two nets.

Whereas female caregiver's regular radio listenership (at least once a week) made no difference in individual member's net use by $15 \%$, regular television viewership (at least once a week) increased the odds by $35 \%$. The female caregiver's low exposure to malaria-related communication messages (one or two messages recalled) did not make a difference compared to no exposure; however, a high level of exposure (three or more messages recalled) was associated with a $45 \%$ percent higher likelihood of household member's net use.

Significant ideational variables for female caregivers included perceived self-efficacy to prevent malaria, perceived self-efficacy to detect malaria, perceived selfefficacy to obtain enough nets for members of her household, perceived response efficacy of nets, descriptive norm about nets, awareness about where to procure nets, and level of participation in decisions regarding net 
Table 1 Results of logistic regression of household members' net use by selected sociodemographic, ideational, and household variables in Madagascar, Mali, and Nigeria

\begin{tabular}{|c|c|c|c|c|c|c|}
\hline \multirow[t]{2}{*}{ Predictor } & \multicolumn{2}{|l|}{ Madagascar } & \multicolumn{2}{|l|}{ Mali } & \multicolumn{2}{|l|}{ Nigeria } \\
\hline & Odds Ratio & Beta $^{a}$ & Odds Ratio & Beta $^{a}$ & Odds Ratio & Beta $^{a}$ \\
\hline \multicolumn{7}{|l|}{ Age group } \\
\hline Under-5 (RC) & 1.00 & - & 1.00 & - & 1.00 & - \\
\hline $5-17$ & $0.411^{* * *}$ & -0.192 & $0.332^{* * *}$ & -0.265 & $0.502^{* * *}$ & -0.162 \\
\hline Adult & 0.934 & -0.016 & $0.374^{* * *}$ & -0.242 & $0.448^{* * *}$ & -0.202 \\
\hline \multicolumn{7}{|l|}{ Gender } \\
\hline Male (RC) & 1.00 & - & 1.00 & - & 1.00 & - \\
\hline Female & 1.155 & 0.034 & $0.839 \neq$ & -0.044 & 1.044 & 0.011 \\
\hline \multicolumn{7}{|c|}{ Age group/Gender Interactions } \\
\hline Under-5 X Male (RC) & 1.00 & - & 1.00 & - & 1.00 & - \\
\hline 5-17 X Female & 0.978 & -0.004 & $1.459^{* * *}$ & 0.073 & 1.084 & 0.015 \\
\hline Adult X Female & $1.282 \ddagger$ & 0.049 & $3.296^{* * *}$ & 0.247 & $1.667^{* * *}$ & 0.107 \\
\hline \multicolumn{7}{|c|}{ Household wealth quintile } \\
\hline Lowest (RC) & 1.00 & - & 1.00 & - & 1.00 & - \\
\hline Second & 0.971 & -0.006 & 0.913 & -0.019 & 0.960 & -0.008 \\
\hline Middle & $0.849 \neq$ & -0.029 & $0.852^{*}$ & -0.031 & 0.962 & -0.008 \\
\hline Fourth & $0.801^{* *}$ & -0.041 & $0.711^{* * *}$ & -0.065 & 0.950 & -0.011 \\
\hline Highest & 0.989 & -0.002 & $0.646^{* * *}$ & -0.088 & $0.879 \neq$ & -0.026 \\
\hline Household size $^{b}$ & & & $0.831^{* * *}$ & -0.316 & & \\
\hline \multicolumn{7}{|l|}{ Household size ${ }^{b}$} \\
\hline $2-4(\mathrm{RC})$ & 1.000 & - & & & & \\
\hline $5-6$ & $0.452^{* * *}$ & -0.173 & & & & \\
\hline $7-8$ & $0.246^{* * *}$ & -0.274 & & & & \\
\hline $9+$ & $0.147^{* * *}$ & -0.338 & & & & \\
\hline \multicolumn{7}{|l|}{ Household size $^{b}$} \\
\hline $2-4(\mathrm{RC})$ & & & & & 1.00 & 1.00 \\
\hline $5-6$ & & & & & $0.529^{* * *}$ & -0.150 \\
\hline $7-9$ & & & & & $0.365^{* * *}$ & -0.234 \\
\hline $10+$ & & & & & $0.207^{* * *}$ & -0.313 \\
\hline \multicolumn{7}{|c|}{ Number of nets in household ${ }^{c}$} \\
\hline $1-2(\mathrm{RC})$ & & & 1.00 & - & & \\
\hline $3-4$ & & & $2.722^{* * *}$ & 0.240 & & \\
\hline 5 or more & & & $5.931^{* * *}$ & 0.415 & & \\
\hline \multicolumn{7}{|c|}{ Number of nets in household ${ }^{c}$} \\
\hline $1-2(\mathrm{RC})$ & 1.00 & - & & & & \\
\hline 3 or more & $2.793^{* * *}$ & 0.218 & & & & \\
\hline \multicolumn{7}{|c|}{ Number of nets in household ${ }^{c}$} \\
\hline $0-1(\mathrm{RC})$ & & & & & 1.00 & - \\
\hline 2 & & & & & $0.893^{* * *}$ & -0.028 \\
\hline 3 or more & & & & & $0.646^{* * *}$ & -0.100 \\
\hline \multicolumn{7}{|l|}{ Zone of residence } \\
\hline Highlands (RC) & 1.00 & - & & & & \\
\hline Sub-desert & $3.595^{* * *}$ & 0.278 & & & & \\
\hline
\end{tabular}


Table 1 Results of logistic regression of household members' net use by selected sociodemographic, ideational, and household variables in Madagascar, Mali, and Nigeria (Continued)

\begin{tabular}{|c|c|c|c|c|c|c|}
\hline \multirow[t]{2}{*}{ Predictor } & \multicolumn{2}{|l|}{ Madagascar } & \multicolumn{2}{|l|}{ Mali } & \multicolumn{2}{|l|}{ Nigeria } \\
\hline & Odds Ratio & Beta $^{a}$ & Odds Ratio & Beta $^{a}$ & Odds Ratio & Beta $^{a}$ \\
\hline Tropical & $2.928^{* * *}$ & 0.231 & & & & \\
\hline Equatorial & $5.076^{* * *}$ & 0.352 & & & & \\
\hline \multicolumn{7}{|l|}{ Region of residence } \\
\hline Koulikoro & & & 1.00 & - & & \\
\hline Sikasso & & & $1.787^{* * *}$ & 0.130 & & \\
\hline Mopti & & & $1.231^{* * *}$ & 0.045 & & \\
\hline Bamako & & & 0.952 & -0.010 & & \\
\hline \multicolumn{7}{|l|}{ State of residence } \\
\hline Akwa Ibom (RC) & & & & & 1.00 & - \\
\hline Kebbi & & & & & $1.487^{* * *}$ & 0.098 \\
\hline Nasarawa & & & & & $1.099 \neq$ & 0.022 \\
\hline \multicolumn{7}{|l|}{ Caregiver's education } \\
\hline Primary or less (RC) & 1.00 & - & 1.00 & - & 1.00 & - \\
\hline Post-primary & 0.925 & -0.011 & $0.849^{* *}$ & -0.032 & 0.957 & -0.010 \\
\hline \multicolumn{7}{|l|}{ Caregiver's religion } \\
\hline Non-Christian (RC) & 1.00 & - & & & 1.00 & - \\
\hline Christian & $0.888 \neq$ & -0.026 & & & $0.865^{*}$ & -0.037 \\
\hline \multicolumn{7}{|l|}{ Caregiver's radio listening habits } \\
\hline Fewer than once a week (RC) & 1.00 & - & 1.00 & - & 1.00 & - \\
\hline At least once a week & 0.911 & -0.022 & 0.977 & -0.006 & 1.024 & 0.006 \\
\hline \multicolumn{7}{|l|}{ Caregiver's TV watching habits } \\
\hline Fewer than once a week (RC) & 1.00 & - & 1.00 & - & 1.00 & - \\
\hline At least once a week & $1.348^{* *}$ & 0.041 & $1.227^{* * *}$ & 0.051 & 1.034 & 0.008 \\
\hline \multicolumn{7}{|l|}{ Caregiver's exposure to messages on malaria } \\
\hline No exposure (RC) & 1.00 & - & 1.00 & - & & \\
\hline Low & 0.975 & -0.006 & 0.962 & -0.009 & & \\
\hline High & $1.447^{* * *}$ & 0.073 & 0.899 & -0.018 & & \\
\hline Heard messages on malaria & & & & & 0.960 & -0.010 \\
\hline \multicolumn{7}{|l|}{ Caregiver's perceived severity of malaria } \\
\hline Lower (RC) & 1.00 & - & 1.00 & - & 1.00 & - \\
\hline Higher & 0.971 & -0.006 & 0.973 & -0.007 & $0.885^{* *}$ & -0.031 \\
\hline Caregiver's perceived susceptibility to malaria & & - & & & & - \\
\hline Lower (RC) & 1.00 & & 1.00 & - & 1.00 & \\
\hline Higher & 0.998 & -0.000 & 0.977 & -0.006 & $0.927 \ddagger$ & 0.019 \\
\hline \multicolumn{7}{|l|}{ Caregiver's perceived self-efficacy to prevent malaria } \\
\hline Lower (RC) & 1.00 & - & 1.00 & - & 1.00 & - \\
\hline Higher & $1.567^{* * *}$ & 0.061 & 1.068 & 0.016 & 1.034 & 0.008 \\
\hline \multicolumn{7}{|c|}{ Caregiver's perceived self-efficacy to detect a serious case of malaria } \\
\hline Lower (RC) & 1.00 & - & 1.00 & - & 1.00 & - \\
\hline Higher & $1.162^{* *}$ & 0.033 & 0.940 & -0.014 & 0.986 & -0.003 \\
\hline \multicolumn{7}{|l|}{ Caregiver's perceived response-efficacy of nets } \\
\hline Lower (RC) & 1.00 & - & 1.00 & - & 1.00 & - \\
\hline
\end{tabular}


Table 1 Results of logistic regression of household members' net use by selected sociodemographic, ideational, and household variables in Madagascar, Mali, and Nigeria (Continued)

\begin{tabular}{|c|c|c|c|c|c|c|}
\hline \multirow[t]{2}{*}{ Predictor } & \multicolumn{2}{|l|}{ Madagascar } & \multicolumn{2}{|l|}{ Mali } & \multicolumn{2}{|l|}{ Nigeria } \\
\hline & Odds Ratio & Beta $^{a}$ & Odds Ratio & Beta $^{a}$ & Odds Ratio & Beta $^{a}$ \\
\hline Higher & 1.137 & 0.017 & 1.060 & 0.012 & $0.919^{*}$ & -0.021 \\
\hline \multicolumn{7}{|c|}{ Caregiver's awareness that fever is a symptom of malaria } \\
\hline Not aware (RC) & 1.00 & - & 1.00 & - & & \\
\hline Aware & 0.985 & -0.003 & $1.155^{* * *}$ & 0.035 & & \\
\hline \multicolumn{7}{|l|}{ Caregiver's awareness that mosquito is the cause of malaria } \\
\hline Not aware (RC) & 1.00 & 1.00 & 1.00 & - & 1.00 & - \\
\hline Aware & 0.982 & -0.003 & $1.365^{* * *}$ & 0.048 & 0.933 & -0.010 \\
\hline \multicolumn{7}{|l|}{ Caregiver's perceived self-efficacy to purchase enough nets } \\
\hline Lower (RC) & 1.00 & - & 1.00 & - & & \\
\hline Higher & $1.344^{* * *}$ & 0.067 & $1.200^{* * *}$ & 0.044 & & \\
\hline Discussed malaria with friends/relations & 1.057 & 0.013 & & & 0.996 & -0.001 \\
\hline Participates in decisions about net allocation & $1.343^{* * *}$ & 0.061 & & & 0.998 & -0.001 \\
\hline \multicolumn{7}{|l|}{ Attitudes towards nets } \\
\hline Negative (RC) & 1.00 & - & & & 1.00 & - \\
\hline Positive & 1.020 & 0.005 & & & 0.968 & -0.008 \\
\hline Perceived net use as the norm among in community & $1.391^{* * *}$ & 0.069 & & & 1.024 & 0.006 \\
\hline Knows where to buy nets & $1.284^{* * *}$ & 0.058 & & & 1.023 & 0.005 \\
\hline Willing to pay for nets & 1.015 & 0.003 & & & 1.045 & 0.011 \\
\hline Pseudo- $R^{2}$ & $17.1 \%$ & & $9.9 \%$ & & $8.6 \%$ & \\
\hline Hosmer-Lemeshow $\mathrm{GOF}^{\mathrm{d}}\left(\boldsymbol{X}^{2} / \mathrm{p}\right)$ & $15.3 / 0.08$ & & $15.5 / 0.08$ & & 14.9/0.09 & \\
\hline Number of Observations & 9260 & & 19,345 & & 15,463 & \\
\hline
\end{tabular}

Notes: $\neq p<0.1 ;{ }^{*} p<0.05 ;{ }^{* *} p<0.01 ;{ }^{* * *} p<0.001$

${ }^{a}$ Fully $(X Y)$ standardized beta coefficients

${ }^{b}$ Household size was not normally distributed in Madagascar and Nigeria. The variable was differently categorized in the two countries in a way that helps to ensure model fit

${ }^{c}$ Number of nets was not normally distributed in any of the study countries. The variable was differently categorized in the three countries in a way that helps to ensure model fit

${ }^{\mathrm{d}}$ GOF Goodness of Fit

allocation within the household. Residents of households where the female caregiver demonstrated perceived selfefficacy to prevent malaria were $57 \%$ more likely to sleep under a net than those in households where the female caregiver did not demonstrate such belief. Similarly, the female caregiver's self-efficacy to detect a severe case of malaria was associated with a $16 \%$ increase in the odds of household member use of a net while the caregiver's perceived self-efficacy to obtain enough nets for her household was associated with a $34 \%$ increase in the odds. Female caregiver's belief that net use was the norm in her community increased the odds of household member's net use by $39 \%$ while her awareness of where to procure a bed net increased the odds by $28 \%$. Finally, members of a household in which the female caregiver participates in net allocation decisions were 34\% more likely to sleep under a net than those in households where the woman did not participate in such decisions.
A look at the magnitude of the fully standardized beta coefficients reveals that the most important predictors of household members' net use were the individual zone of residence, number of nets in the household, and household size and age, in order of importance. Among the female caregiver's ideational variables, the most important were descriptive norms (perceptions about how other people behave), perceived self-efficacy to procure enough nets for the household, participation in household net allocation decisions, perceived self-efficacy to prevent malaria, and awareness of where to procure nets.

Figure 2 shows a graduated, dose-response relationship of net use with ideation score, controlling for all other non-ideational variables. The percent who slept under a net on the night before the survey increased from 54.5\% in households where the female caregiver had zero positive ideational variables (out of six) to $83.2 \%$ in households where the female caregiver had all six positive 


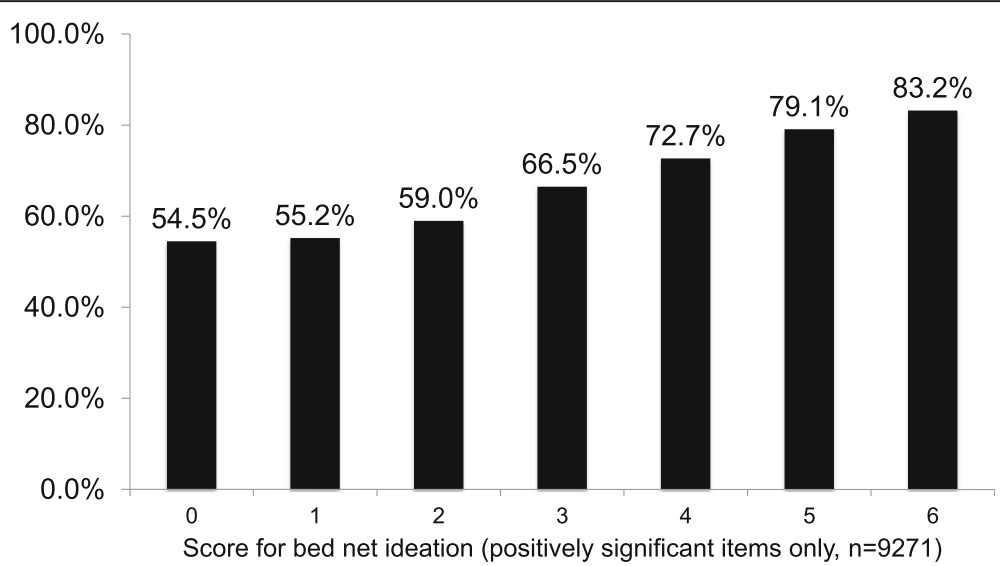

Fig. 2 Predicted probability of household bed net use, by female caregiver's cumulative ideation score, Madagascar 2014. Detailed legend: Probability of household bed net use as a function of female caregivers' cumulative ideation score in Madagascar 2014 (range $=0-6$, mean = 4.13, SD = 1.18)

ideational variables. When the composite ideation score (mean $=4.13, \mathrm{SD}=1.18$ ) was substituted for the individual ideational variables in a logistic regression, the results indicate a strong positive relationship, controlling for all other non-ideational variables. Specifically, a onepoint increase in the caregiver's ideation score increases the household member's odds of sleeping under a net by about $37 \%$.

\section{Mali \\ Bivariate analysis}

In Mali, possession of nets was almost universal in 2014-2015: only $3.1 \%$ of the households in the study regions did not have at least one bed net. Net use information was available for 19,345 household members (9436 males and 9909 females). The majority of household members $(82.1 \%)$ slept under a net on the night preceding the survey. Differences by gender, age group, and region of residence were significant. More than four-fifths (85.3\%) of females compared to $78.7 \%$ of males slept under a net on the night before the survey $(\mathrm{z}=14.2 ; p<$ 0.001 ). Net use was less prevalent among older children aged 5-17 years (76.2\%) compared to children under five $(88.4 \%)$ and adults $(84.1 \%) \quad\left(\mathrm{X}^{2}=298.3 ; p<0.001\right)$. Variations by region of residence were also noticeable, varying from $78.5 \%$ in Koulikoro to $87.3 \%$ in Sikasso $\left(\mathrm{X}^{2}\right.$ $=155.4 ; p<0.001$ ).

\section{Multivariate analysis}

Results of the multivariate logistic regression revealed that the predictors of net use in Mali operate at various levels and include age, gender, number of nets in the household, and region of residence (Table 1). Other significant demographic predictors include household wealth, household size, and level of education of the female caregiver.
Among males, the odds of net use were significantly lower among boys aged 5-17 years (67\% lower) and men (63\% lower) compared to children under five. Among children under five, there was no difference between boys and girls in the odds of net use. The association of age with net use depends on gender: being female attenuates the negative relationship of bed net use with age. There was a graduated reduction in the odds of net use across wealth quintiles such that the odds of net use were about $35 \%$ less in households in the highest quintile and $15 \%$ less in households in the middle quintile compared to the poorest households. The relationship of net use with household size was negative: each additional household member decreased the odds of net use by $17 \%$. In contrast, the relationship with the number of nets was positive: people in households with three or four nets were almost three times as likely and those in households with five or more nets were almost six times as likely to use a net compared to their peers in households with one or two nets. Whereas there was no difference between Koulikoro and Bamako, the residents of Sikasso and Mopti were significantly more likely to use a net compared to their peers in Koulikoro.

The association of net use with caregiver's education was negative in Mali, with members of households where the woman has post-primary education being about $15 \%$ less likely to use a net compared to their peers in households where the woman had primary education or less. Female caregiver's regular radio listening was not associated with net use, but regular television viewing increased the odds of household members' net use by $23 \%$. The caregiver's exposure to malaria-related messages made no significant difference in net use independently of what was attributable to general media habits.

In terms of ideational variables, there was a strong positive association with caregiver's awareness of fever as a symptom of malaria and mosquito as the cause of malaria. Similarly, the relationship with caregiver's perceived self- 
efficacy to obtain enough nets for members of her family was positive and significant. Rather surprisingly, household members' net use was negatively associated with the female caregiver's perceived self-efficacy to detect a severe case of malaria.

Examination of fully standardized beta coefficients revealed that the most important determinants of household members' net use were age, household size, and number of nets. The most important caregiver's ideational variables were her malaria-related knowledge and her perceived self-efficacy to obtain enough nets for members of her family.

The multivariable logistic regression that included the overall ideation score $($ mean $=2.73, \mathrm{SD}=1.08)$ in place of the individual ideational variables revealed a dose response relationship between caregiver's ideation score and household members' net use. For example, the odds of household member net use was more than twice (2. 06) as high in households where the female caregiver scored three out of three on the positive ideation score compared to households where the female caregiver scored zero out of three.

\section{Nigeria}

\section{Bivariate analysis}

In Nigeria, only one-third (33.7\%) of residents of households with at least one net slept under a net. Among these households, net use varied significantly by state of residence: $32.1 \%$ in Akwa Ibom compared to $38.2 \%$ in Kebbi and $29.9 \%$ in Nasarawa $\left(\mathrm{X}^{2}=92.6 ; p<0.001\right)$. There were also significant differences by age: $44.7 \%$ of children under 5 years old slept under a net compared to $24.4 \%$ among children age $5-17$ years and $32.9 \%$ of adults $\left(\mathrm{X}^{2}=463.3 ; p<0.001\right)$. There were also significant differences by gender with net use being more common among females (36.2\%) than males (31.1\%) $\left(\mathrm{X}^{2}=48,1 ; p\right.$ $<0.001)$.

\section{Multivariate analysis}

Results of the multivariate logistic regression indicate that the significant predictors of net use among household members are household size, age, state of residence, number of nets, and being of Christian faith, in order of magnitude. Among males, compared to children under five, 5-17 year olds (50.2\% lower) and adults (55.2\% lower) were significantly less likely to have slept under a net. There was no significant sex difference among children under five. When looking at the interaction between age and gender, being female significantly improved the odds of adult net use. Unsurprisingly, as household size increased, odds of using a net decreased. For example, the likelihood of net use was about $79 \%$ lower for people from households with ten or more members compare to those from households with fewer than five members. There was a negative dose response association between net use and number of nets (a 12\% decrease in likelihood), which is opposite of what we found in Madagascar and Mali. The odds of using a net were significantly higher in Kebbi (49\%), but not in Nasarawa or Akwa Ibom. Respondents of Christian faith were $15 \%$ less likely to use a net than those practicing other religions.

Use of radio or television and reported level of exposure to malaria messages by the female caregiver were not significant predictors of net use in Nigeria. In terms of ideational variables, there was a negative relationship between net use and caregiver's perceived severity of malaria, with the odds of net use being $12 \%$ lower among members of households where the caregiver has a higher level of perceived severity of malaria compared to their peers in household where the caregiver has a lower level of perceived severity. There was also a negative relationship between caregiver's perceived response efficacy of nets and household members' net use. No other ideational variables were significant in Nigeria.

\section{Discussion}

The ideation model of communication and behavior change has been used effectively in a number of health domains to guide the strategic design and evaluation of health communication interventions, but it has received relatively little attention in malaria prevention efforts. This study attempted to compare the dynamics of ideation and behavior in three countries in order to determine how it differs by context. Results indicate that a more diverse set of ideational factors play a role in household bed net use in Madagascar compared to Mali or Nigeria, and that the strongest ideational predictors of bed net use vary from one country to another, although efficacy-related variables appear to be important in all three settings. In our view, this demonstrates the usefulness of ideation as a conceptual tool that can help researchers and program planners to identify common psychosocial variables and systematically examine how they influence malaria-related behavior in a particular programmatic context, controlling for prevailing local conditions.

\section{The role of independent demographic variables}

In terms of demographic predictors of bed net use, there were more similarities than differences across the three countries analyzed in this study, even though Madagascar and Mali had much higher rates of bed net use $(73 \%$ and $82 \%$, respectively) compared to Nigeria (34\%). In all three countries, children under 5 years old were more likely to sleep under a bed net compared to older children aged 517 years (and adults, except in Madagascar). Also in all three countries, the odds of bed net use were lower on 
average in larger households. The documented relationships with age and household size are consistent with what other studies have found in Africa and elsewhere [14, 57-60]. Also in Madagascar and Mali, bed net use was higher in the poorest households compared to wealthier households. Only in Mali were families whose caregivers had lower levels of education significantly more likely to use bed nets than families whose caregivers had more education. In Nigeria, Christians were less likely to use bed nets than Muslims. Findings of this nature can help programmers to target subgroups that are at higher risk.

In Madagascar and Mali, households with caregivers who regularly watched television were more likely to report bed net use. Consistent with findings from other studies, in Madagascar, caregivers' higher malaria message recall was associated with higher rates of bed net use $[4,14,16,61]$. The reasons for the lack of significant association between caregiver's exposure to malaria messages and bed net use in Mali and Nigeria were not clear; we know little about the intensity or content of malaria messages except in Nigeria, so it is hard to attribute the findings to the reach or quality of the information provided. The overall lower use of bed nets in Nigeria may result in lower salience of malaria messaging, which would reduce motivations to pay attention to and process malaria information, even though communication campaigns were underway.

\section{Effects of independent ideational variables}

In terms of ideational predictors of bed net use, multiple factors were positively correlated with net use in Mali and Madagascar. Counterintuitively, in Nigeria, the higher the caregiver's perceived severity of a malaria infection or her perceived response efficacy of nets (i.e., belief that bed net use can prevent infection), the lower the reported bed net use. Some conceptual frameworks that focus on threat and efficacy as predictors of behavior see high perceived risk as potentially debilitating-a frightened person may not take action to counter a disease threat if there is no accompanying belief in one's ability to manage the threat [27, 62-64]. Sometimes selfefficacy can result in lower rates of behavior if it takes the form of overconfidence to control threats and achieve health outcomes [65-68]. This may be the case in Nigeria, where there was no evidence that selfefficacy perceptions-self-efficacy to detect or prevent malaria or self-efficacy to obtain bed nets-were associated with bed net use. People may feel so confident in their ability to detect and treat a severe case of malaria that they feel less threatened by the disease, and are therefore less motivated to sleep under a net.

In Mali, consistent with findings from many previous studies, knowledge about malaria played a role in motivating behavior: people who knew that fever is a symptom of malaria and who knew that mosquitoes spread malaria were more likely to report bed net use [57, 69, 70]. This finding was in contrast to what we found for the other two study countries. Without more information about existing malaria communication in Mali, it is difficult to know why these differences exist. Some malaria programs in Sub-Saharan Africa emphasis rapid careseeking in response to fever as part of large scale "test and treat" campaigns. Such programs would draw attention to information about malarial fever caused by mosquitos versus non-malarial fever and the importance of being tested to know the difference. In such a case, a stronger association between mosquitos and fever could result in greater interest in bed net use to prevent both. Future analysis of how malaria campaigns with different emphases may reinforce (or interfere with) each other would be useful.

In Madagascar, self-efficacy to prevent malaria and self-efficacy to obtain bed nets were associated with higher reported net use, echoing findings from other studies, including a similar study conducted in Liberia [14]. Unlike Mali, in Madagascar self-efficacy to detect a severe case of malaria was positively associated with net use. Also in Madagascar three additional ideational variables were positive predictors of bed net use: the belief that bed net use was the community norm, knowing where to obtain a bed net, and participation at the household level in decision-making about who would sleep under a bed net. Why more ideational variables were significant in Madagascar compared to Mali or Nigeria is not clear. Greater familiarity or experience with an issue is often associated with greater cognitive elaboration; if malaria communication (whatever its content) is more intensive or far-reaching in one place compared to another, that could explain more nuanced perceptions about disease threat and prevention. On the other hand, where exposure to information has been low, the introduction of an information campaign can have novelty effects-people learn a lot very quicklywhereas familiarity with an issue sometimes results in more counterarguing and rejection of new information. More research on the history and content of malaria communication in these countries would be needed to determine which explanation is more likely.

As summarized in Table 2, of the 14 ideational variables examined in this study, six were significant predictors of bed net use in Madagascar (all positively), four were significant predictors of bed net use in Mali (all positively), and only two were significant predictors in Nigeria (both negatively).

Madagascar and Mali share only one of the ideational predictors: self-efficacy to obtain enough bed nets for your household. In Nigeria, perceived severity of malaria and perceived response efficacy of nets were the only 
Table 2 Summary of ideational predictors of bed net use, by country

\begin{tabular}{|c|c|c|c|}
\hline \multirow[t]{2}{*}{ Predictor } & \multicolumn{3}{|l|}{ Country } \\
\hline & Madagascar & Mali & Nigeria \\
\hline Perceived severity of a malaria infection & ns & ns & - \\
\hline Self-efficacy to detect severe case of malaria & + & ns & ns \\
\hline Self-efficacy to prevent malaria & + & ns & ns \\
\hline Self-efficacy to obtain enough nets for the household & + & + & ns \\
\hline Response efficacy (belief that nets are effective) & ns & ns & - \\
\hline Perceived community norm of bed net use & + & na & ns \\
\hline Knowledge of where to get bed nets & + & na & ns \\
\hline Knowledge that fever is a sign of malaria & ns & + & ns \\
\hline Knowledge that mosquitos are the malaria vector & ns & + & ns \\
\hline Participating in decision-making about net allocation & + & na & ns \\
\hline Number of positively significant ideational predictors of bed net use & 7 & 3 & 0 \\
\hline
\end{tabular}

Key: + (positively relationship), - (negatively significant), ns (not significant), na (not measured)

ideational variables associated (negatively) with bed net use. This finding suggests that structural and demographic barriers as well as psychosocial influences may need to be addressed as determinants of bed net use in that country.

It is important to note that another study in Nigeria that included measures of female caretakers' malariarelated ideation [71] found it to be significantly associated with the caretaker's own use of a bed net, rather than bed net use by members of her household. Other factors may also explain why household bed net use is more weakly correlated with caretaker's ideation in Nigeria. For example, it may be that female caregivers in Nigeria have less agency to influence bed net use by other members of the household compared to female caregivers in Mali and Madagascar, thus weakening the causal link between caregiver ideation and household member behavior.

\section{Implications for effective malaria social and behavior change strategies}

It is essential to note that age, region, household size, and number of nets in the household were the strongest predictors of bed net use, demonstrating that families are prioritizing younger children, consistent with recent studies [72], and that the primary barriers to bed net use are still largely due to access. Controlling for these structural barriers permits SBCC programs to then isolate pertinent ideational variables. Of all the ideational variables examined in these three countries, those that were the most consistently significant predictors of bed net use were related to efficacy perceptions: self-efficacy to obtain bed nets for your household and self-efficacy to detect malaria. This suggests that efficacy-based communication strategies are likely to produce desirable results. Programs should emphasize increasing and reinforcing the head of household's and caregiver's confidence in responding to the threat of malaria and their correct and consistent net use as a preventive measure. The widely validated social learning approach of modeling effective behavior [21] could be used to show or describe locally acceptable and identifiable examples of effective net use and other preventive behaviors in an effort to build local self-confidence in malaria prevention. Showing how nets can be obtained and used may be particularly important in places like Nigeria where net use and the access to use ratio are low to begin with, and might complement expanded distribution efforts and accelerate uptake. Finally, while we cannot confirm the relationship between female agency and decision making using the current datasets, it may be important in settings where caregivers have less household authority to influence decision makers or other female caregivers in the same household. In such settings, modeling efficacious communication in the household about bed net use could be beneficial.

This study also provides support for the hypothesis of a cumulative impact of ideational variables. In Madagascar and Mali, where multiple ideational variables were significant predictors of bed net use (controlling for the effect of household and sociodemographic variables), bed net use was higher when more ideational variables were positive. This suggests that it may be effective to bundle more than one ideational variable into program messaging, for example, by addressing multiple aspects of self-efficacy and malaria knowledge in an integrated communication strategy.

\section{Limitations of this study}

The use of bed nets by household members was determined through an enumeration of household residents, 
of the nets available in the household, and through subsequent questions about who slept under each of those nets on the night prior to the survey. This produced an outcome variable representing whether an individual slept under a net the night before the survey. Yet, data on ideational variables were collected only from the female caregivers of a selected child under 5 years old in each household. Our analysis assumes that what a particular female caregiver-who may be only one of several caregivers in the household-knows, thinks, and feels about malaria and bed net use will have an impact on the bed net use patterns of the entire household. Ultimately, bed net use is a volitional behavior, particularly for older children and adults who may have the power to make their own choices and are therefore influenced by their own individual attitudes, beliefs, and preferences as opposed to those of the female caregiver who was interviewed. While our data indicate that there is a positive relationship between female caregiver ideation and household net use, controlling for numerous other household and demographic variables, the actual causal pathways are undoubtedly more complicated. Future studies may wish to collect ideational data from more than one individual in a household or, perhaps, include more questions about the content of interpersonal discussion of malaria prevention and net use among household members, in order to better understand the broader household context of net use decisions.

\section{Conclusion}

While the strongest predictors of net use among households with at least one bed net were age, region, household size, and number of nets, this study has demonstrated the importance of controlling for these variables when trying to identify the ideational variables that influence caregiver bed net use in three African countries, because the relevant ideational variables varied across countries. In Madagascar, the two most significant ideational predictors of bed net use were the caregiver's descriptive norm about bed net use and her awareness of where to procure a bed net. In Mali, the most important caregiver's ideational variables were her perceived self-efficacy for obtaining bed nets and her malaria-related knowledge. While no ideational variable was positively linked with bed net use in Nigeria, this finding calls for additional study to identify other possible social, structural, and supply-side explanations for bed net use.

In short, this research suggests that integrating more than one ideational variable-such as malaria knowledge and one or more aspects of self-efficacy-into program messaging, could result in a more effective integrated communication strategy.

\section{Abbreviations}

HC3: Health Communication Capacity Collaborative; IRB: Institutional Review Board; ITN: insecticide-treated bed net; PMI: U.S. President's Malaria Initiative; SBCC: social and behavior change communication; USAID: United States Agency for International Development

\section{Acknowledgments}

This study was made possible by the generous support of the American People through the United States Agency for International Development (USAID) and the U.S. President's Malaria Initiative (PMI). The Health Communication Capacity Collaborative (HC3) is based at Johns Hopkins Center for Communication Programs and supported by USAID's Bureau for Global Health under Cooperative Agreement \#AID-OAA-A-12-00058. The contents of this report are the sole responsibility of the authors and does not necessarily represent the views or positions of USAID, PMI, the US Government, or The Johns Hopkins University. The authors would like to thank Andrew Tompsett and Don Dickerson from USAID for their support of the activities that led to the development of this manuscript and for their insightful feedback on the manuscript.

\section{Funding}

The work was funded through USAID's Bureau for Global Health under Cooperative Agreement \#AID-OAAA-12-00058. The funders did not influence the study design, data analysis, or interpretation of results.

\section{Availability of data and materials}

The datasets used and/or analysed during the current study are available from the corresponding author on reasonable request.

\section{Authors contributions \\ JDS was the lead author and editor of the manuscript, helped design the studies and data analysis and was Acting Research Director of the HC3 project at the time these surveys were implemented. SOB was Principal Investigator for the three surveys, lead the data analysis and contributed major portions of the manuscript on research methods, data analysis and results. EER provided data analysis support and contributed to the methods and results sections. KAF provided editorial support throughout the manuscript preparation process. MT and NL provided programmatic support during the implementation of the surveys and participated with JDS, SOB, and EER in meetings with the President's Malaria Initiative that helped to shape the objectives of the study design and interpretation of the results. JDS, SOB, KAF, MT, NL, and HK provided technical guidance, and revised and reviewed drafts of this manuscript. All authors read and approved the final manuscript.}

\section{Ethics approval and consent to participate}

Ethical clearance for all three surveys was obtained from The Johns Hopkins Bloomberg School of Public Health Institutional Review Board (IRB) and local IRB in Madagascar (Ministry of Health), Mali (Institut National de Recherche en Santé Publique), and Nigeria (National Health Research Ethics Committee). In all three countries, interviewers obtained oral informed consent from participants prior to the interviews. Written consent is uncommon for minimal risk studies in all three countries for two reasons: (1) literacy levels are generally low and (2) no personal identifiers were collected for the survey, so a participant's signature on a consent form would represent an unnecessary identifier that could link a participant to her/his data. All three local IRBs approved this process.

\section{Consent for publication}

Not applicable

\section{Competing interests}

The authors declare that they have no competing interests.

\section{Publisher's Note}

Springer Nature remains neutral with regard to jurisdictional claims in published maps and institutional affiliations. 


\section{Received: 30 October 2017 Accepted: 26 March 2018}

\section{Published online: 11 April 2018}

\section{References}

1. World Health Organization. World malaria report 2016. Geneva: World Health Organization; 2016.

2. Alba S, Nathan R, Schulze A, Mshinda H, Lengeler C. Child mortality patterns in rural Tanzania: an observational study on the impact of malaria control interventions. Int J Epidemiol. 2014;43:204-15.

3. Lengeler $C$. Insecticide-treated bed nets and curtains for preventing malaria. Cochrane Database Syst Rev. 2004;2:CD000363. http://onlinelibrary.wiley. com/doi/10.1002/14651858.CD000363.pub2/abstract;jsessionid= 23CE1FBBFA0C06D2841A1BBC3EE93F02.f02t02. Accessed 28 Mar 2018.

4. Ricotta EE, Boulay M, Ainslie R, et al. The use of mediation analysis to assess the effects of a behavior change communication strategy on bed net ideation and household universal coverage in Tanzania. Malar J. 2015;14:15.

5. Roll Back Malaria Vector Control Working Group. Continuous distribution Workstream. Consensus statement on continuous distribution Systems for Insecticide Treated Nets. Geneva: Roll Back Malaria Partnership; 2011. https:// www.vector-works.org/wp-content/uploads/vcwg6report1.pdf. Accessed 28 Mar 2018

6. White MT, Conteh L, Cibulskis R, Ghani AC. Costs and cost-effectiveness of malaria control interventions - a systematic review. Malar J. 2011;10:337.

7. Yukich JO, Lengeler C, Tediosi F, et al. Costs and consequences of largescale vector control for malaria. Malar J. 2008;7:258.

8. Eisele TP, Keating J, Littrell M, Larsen D, Macintyre K. Assessment of insecticide-treated bednet use among children and pregnant women across 15 countries using standardized national surveys. Am J Trop Med Hyg. 2009;80:209-14

9. Hetzel MW, Gideon G, Lote N, Makita L, Siba PM, Mueller I. Ownership and usage of mosquito nets after four years of large-scale free distribution in Papua New Guinea. Malar J. 2012;11:192.

10. Koenker H, Ricotta E. Insecticide-treated nets access and use report. Johns Hopkins Center for Communication Programs: Baltimore; 2016. http://www. vector-works.org/resources/itn-access-and-use/. Accessed 28 Mar 2018.

11. Global Malaria Programme. Achieving universal coverage with long-lasting insecticidal nets in malaria control. Recommendation. Rev. 1. Geneva: World Health Organization; 2014. http://www.who.int/malaria/publications/atoz/ who_recommendations_universal_coverage_Ilins.pdf. Accessed 28 Mar 2018.

12. Koenker $\mathrm{H}$, Kilian A. Recalculating the net use gap: a multi-country comparison of ITN use versus ITN access. PLoS One. 2014;9(5):e97496.

13. Adjah ESO, Panayiotou AG. Impact of malaria related messages on insecticidetreated net (ITN) use for malaria prevention in Ghana. Malar J. 2014;13:123.

14. Babalola S, Ricotta E, Awantang G, Lewicky N, Koenker H, Toso M. Correlates of intra-household ITN use in Liberia: a multilevel analysis of household survey data. PLoS One. 2016;11(7):e0158331.

15. Bowen HL. Impact of a mass media campaign on bed net use in Cameroon. Malar J. 2013;12(1):36.

16. Kilian A, Balayo C, Feldman M, et al. The effect of single or repeated home visits on the hanging and use of insecticide-treated mosquito nets following a mass distribution campaign - a cluster randomized, controlled trial. PLoS One. 2015;10(2):e0119078.

17. Fishbein M, Triandis HC, Kanfer FH, Becker M, Middlestadt SE. Factors influencing behavior and behavior change. In: Baum AS, Revenson TA, Singer JE, editors. Handbook of health psychology. Mahwah: Lawrence Erlbaum; 2001. p. 1-17.

18. Johns Hopkins Center for Communication Programs. Health communication partnership: research 101 - a primer for health communication professionals: ideation. Baltimore: Johns Hopkins Center for Communication Programs; 2004.

19. Kincaid DL. Mass media, ideation, and behavior: a longitudinal analysis of contraceptive change in the Philippines. Communic Res. 2000;27(6):723-63.

20. Kincaid DL. Social networks, ideation, and contraceptive behavior in Bangladesh: a longitudinal analysis. Soc Sci Med. 2000;50(2):215-31.

21. Bandura A. Social foundations of thought and action: a social cognitive theory. Englewood Cliffs: Prentice Hall; 1986.

22. Bandura A. Social cognitive theory: an agentic perspective. Asian J Soc Psychol. 1999;2:21-41.

23. Fishbein $\mathrm{M}$, Ajzen I. Belief, attitude, intention, and behavior: an introduction to theory and research. Reading: Madison-Wesley; 1975.
24. McGuire WJ. Theoretical foundations of campaigns. In: Rice RR, Atkin CK editors. Public communication campaigns. 2nd ed. Newbury Park: Sage; 1989. p. 41-70.

25. Prochaska JO, DiClemente CC, Norcross JC. In search of how people change: applications to addictive behaviors. Am Psychol. 1992;47(9):1102-12.

26. Clark MS, editor. Emotion and social behavior. Review of personality and social psychology, vol. 14. Thousand Oaks: Sage; 1992.

27. Witte K. Message and conceptual confounds in fear appeals: the role of threat, fear and efficacy. South Commun J. 1993;58:147-55.

28. Zajonc RB. On the primacy of affect. Am Psychol. 1984;39(2):117-23.

29. Festinger L. A theory of social comparison processes. Hum Relat. 1954;7(2): 117-40.

30. Kincaid DL. Communication network dynamics: cohesion, centrality, and cultural evolution. In: Richards Jr WD, Barnett GA, editors. Progress in communication sciences, vol. 12. Norwood: Ablex; 1993. p. 111-33.

31. Latané B. The psychology of social impact. Am Psychol. 1981;36(4):343-65.

32. Montgomery MR, Casterline JB. Social learning, social influence, and new models of fertility. Popul Dev Rev. 1996;22:151-75.

33. Moscovici S. Social influence and conformity. In: Lindzey G, Aronson E, editors. Handbook of social psychology, vol. 2. New York: Random House; 1986. p. 347-412

34. Rogers EM, Kincaid DL. Communication networks: toward a new paradigm for research. New York: Free Press; 1981.

35. Suls JM. Social comparison theory and research. In: Suls JM, Miller RL, editors. Social comparison processes. New York: Wiley; 1977. p. 1-8.

36. Babalola S, Vondrasek C. Communication, ideation and contraceptive use in Burkina Faso: an application of the propensity score matching method. J Fam Plann Reprod Health Care. 2005;31(3):207-12.

37. MEASURE Evaluation, MEASURE DHS, President's Malaria Initiative, Roll Back Malaria Partnership, UNICEF, World Health Organization. Household survey indicators for malaria control. Chapel Hill: MEASURE Evaluation; 2013.

38. Babalola S, Folda L, Babayaro $H$. The effects of a communication program on contraceptive ideation and use among young women in northern Nigeria. Stud Fam Plan. 2008:39(3):211-20.

39. Kincaid DL, Storey JD, Figueroa ME, Underwood CR. Communication, ideation and contraceptive use: the relationships observed in five countries (unpublished). Proceedings of the world congress on communication for development. Washington: World Bank; 2007.

40. Krenn S, Cobb L, Babalola S, Odeku M, Kusemiju B. Using Nigerian urban reproductive health initiative. Glob Health Sci Pract. 2014;2(4):427-43.

41. Babalola S. Readiness for HIV testing among young people in northern Nigeria: the roles of social norm and perceived stigma. AIDS Behav. 2007; 11(5):759-69.

42. Bertrand JT, O'Reilly K, Denison J, Anhang R, Sweat M. Systematic review of the effectiveness of mass communication programs to change HIV/AIDS-related behaviors in developing countries. Health Educ Res. 2006;21(4):567-97.

43. Naugle DA, Hornik RC. Systematic review of the effectiveness of mass media interventions for child survival in low- and middle-income countries. J Health Commun. 2014;19(Suppl 1):190-215.

44. Figueroa ME, Kincaid DL. Household water treatment in Guatemala: the influence of ideational factors on sustained practice. In: Key summary of findings from the Guatemala water survey, 2003. Baltimore: Johns Hopkins Center for Communication Programs; 2004.

45. Figueroa ME, Kincaid DL. Social, cultural and behavioral correlates of household water treatment and storage. Baltimore: Johns Hopkins Center for Communication Programs; 2010.

46. Underwood C, Schwandt H. Community support and adolescent girls' vulnerability to HIV/AIDS: evidence from Botswana, Malawi, and Mozambique. Int Q Community Health Educ. 2015;35(4):317-34.

47. Figueroa ME. A theory-based socioecological model of communication and behavior for the containment of the Ebola epidemic in Liberia. J Health Commun. 2017;22(Suppl 1):5-9.

48. Norman DA, Verganti R. Incremental and radical innovation: design research vs. technology and meaning change. Design Issues. 2014;30(1):78-96.

49. Institut National de la Statistique (INSTAT), Programme National de lutte contre le Paludisme (PNLP), Institut Pasteur de Madagascar (IPM), ICF International. Enquête sur les Indicateurs du Paludisme (EIPM) 2013. Calverton: INSTAT, PNLP, IPM, ICF International; 2013.

50. National Malaria Elimination Programme (NMEP), National Population Commission (NPopC), National Bureau of Statistics, ICF International. Nigeria 
malaria Indicator survey 2015: key indicators. Abuja; Rockville: NMEP, NPopC, ICF International; 2016.

51. Programme National de Lutte contre le Paludisme (PNLP), Institut National de la Statistique (INSTAT), INFO-STAT, Institut National de la Recherche en Santé Publique (INRSP), ICF International. Enquête sur les Indicateurs du Paludisme au Mali (EIPM) 2015. Rockville: INSTAT, INFO-STAT, ICF International; 2016.

52. U.S. President's Malaria Initiative, Nigeria Country Profile https://www.pmi. gov/docs/default-source/default-document-library/country-profiles/nigeria_ profile.pdf?sfvrsn=18. Accessed 28 Mar 2018.

53. PNLP, MRTC and INFORM. An epidemiological profile of malaria in Mali. UK: A report prepared for the Ministry of Health, Mali, the Roll Back Malaria Partnership and the Department for International Development; 2015.

54. Kesteman T, Randrianarivelojosia M, Mattern C, et al. Nationwide evaluation of malaria infections, morbidity, mortality, and coverage of malaria control interventions in Madagascar. Malar J. 2014;13:465.

55. Kilian $\mathrm{A}$, Lawford $\mathrm{H}$, Ujuju $\mathrm{CN}$, et al. The impact of behaviour change communication on the use of insecticide treated nets: a secondary analysis of ten post-campaign surveys from Nigeria. Malar J. 2016;15:422.

56. Roll Back Malaria Monitoring and Evaluation Reference Group Survey and Indicator Task Force. Household survey indicators for malaria control. Chapel Hill: MEASURE Evaluation; 2013.

57. Biadgilign S, Reda A, Kedir H. Determinants of ownership and utilization of insecticide-treated bed nets for malaria control in eastern Ethiopia. J Trop Med. 2012;2012:235015.

58. Ng'ang'a PN, Jayasinghe G, Kimani V, et al. Bed net use and associated factors in a rice farming community in Central Kenya. Malar J. 2009;8:64.

59. Nuwaha F. Factors influencing the use of bed nets in Mbarara municipality of Uganda. Am J Trop Med Hyg. 2001;65(6):877-82.

60. Panter-Brick C, Clarke SE, Lomas H, Pinder M, Lindsay SW. Culturally compelling strategies for behavior change: a social ecology model and case study in malaria prevention. Soc Sci Med. 2006;62(11):2810-25.

61. Jombo GTA, Mbaawuaga EM, Gyuse AN, et al. Socio-cultural factors influencing insecticide treated bed net utilization in a malaria endemic city in north-Central Nigeria. Asian Paci. J Trop Med. 2010;3(5):402-6.

62. Rogers RW. A protection motivation theory of fear appeals and attitude change1. Aust J Psychol. 1975;91(1):93-114.

63. Rogers RW. Cognitive and physiological processes in fear appeals and attitude change: a revised theory of protection motivation. In: Cacioppo JT, Petty RE, editors. Social psychophysiology: a sourcebook. New York: Guilford Press; 1983. p. 153-76.

64. Rogers RW, Prentice-Dunn S. Protection motivation theory. In: Gochman DS, editor. Handbook of health behavior research I: personal and social determinants, vol. 1. New York: Plenum Press; 1997. p. 113-32.

65. Moore DA, Healy PJ. The trouble with overconfidence. Psychol Rev. 2008; 115(2):502.

66. Pajares F. Current directions in self-efficacy research. Adv Motiv Achiev. 1997:10(149):1-49.

67. Stone DN. Overconfidence in initial self-efficacy judgments: effects on decision processes and performance. Organ Behav Hum Decis Process. 1994;59(3):452-74.

68. Vancouver JB, Kendall LN. When self-efficacy negatively relates to motivation and performance in a learning context. J Appl Psychol. 2006;91(5):1146.

69. Esimai OA, Aluko OO. Determinants of use of insecticide treated bednets among caregivers of under five children in an urban local government area of Osun state, south-western Nigeria. Glob J Health Sci. 2014;7(2):20-7.

70. García-Basteiro AL, Schwabe C, Aragon C, et al. Determinants of bed net use in children under five and household bed net ownership on Bioko Island, Equatorial Guinea. Malar J. 2011;10:179.

71. Babalola S, Okoh M, Berg K. Household sample survey on ideation and behaviors related to malaria prevention and treatment in Nigeria, 2015. Technical report. Baltimore: Johns Hopkins Center for Communication Programs; 2016.

72. Ricotta E, Koenker H, Kilian A, Lynch M. Are pregnant women prioritized for bed nets? An assessment using survey data from 10 African countries. Glob Health Sci Pract. 2014;2(2):165-72.

73. Kincaid DL, Delate R, Storey JD, Figueroa ME. Advances in Theory Driven Design and Evaluation of Health Communication Campaigns: Closing the Gap in Practice and Theory. In: Rice R, Atkin C, editors. Public Communication Campaigns, 4th Ed. Newbury Park: Sage; 2012. p. 305-319.

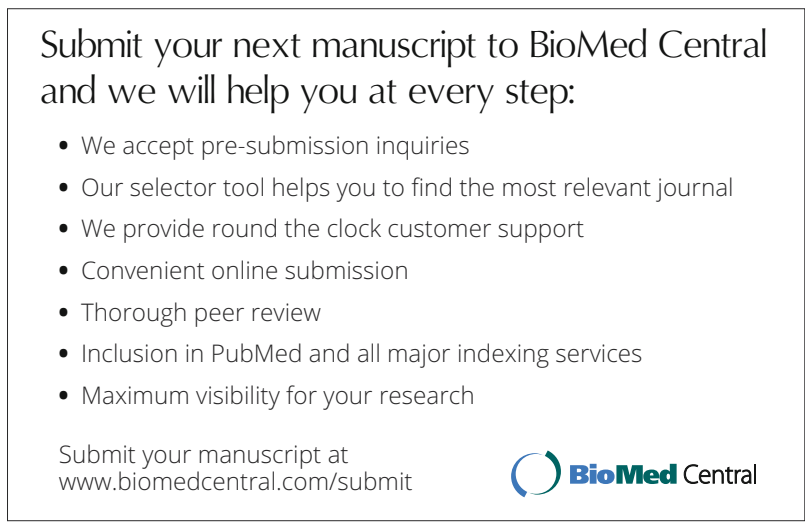

